\title{
Measuring memory for source: Some theoretical assumptions and technical limitations
}

\author{
KEVIN MURNANE \\ University of Maryland, College Park, Maryland \\ and \\ UTE J. BAYEN \\ University of North Carolina, Chapel Hill, North Carolina
}

\begin{abstract}
Henkel and Franklin (1998) present a series of well-designed experiments in support of the conclusion that memory for the source of an item is affected by the similarity between the item and other information in memory. Their principle analyses use an empirical measure of source memory that is a variant of a measure evaluated by Murnane and Bayen (1996). We point out an important assumption that underlies the use of this measure and question additional arguments and analyses that Henkel and Franklin offer in support of their conclusions. The problems discussed illustrate the need for careful consideration of the technical characteristics of measures and the theoretical assumptions on which measures rest when one is conducting research on source memory.
\end{abstract}

In this commentary, we discuss several questions concerning the measurement of source memory that arise from the data analyses reported by Henkel and Franklin (1998). Henkel and Franklin's findings have important theoretical implications, their experiments are carefully designed, their exposition is clear, and they have used the best available empirical measure to measure source memory in their experiments. Although we disagree with some of what we find in Henkel and Franklin, we offer this commentary more to make what we believe are important points about the measurement of source memory in general than to criticize Henkel and Franklin's research.

Although measurement theory does not often attract a great deal of attention, everyone would agree that measurement is important. Many of the dependent measures that are often used in memory research, such as the probability of a correct response in a recall task, or $d^{\prime}$ as a measure of discrimination in a recognition task, have been carefully analyzed and are well understood. It is easy to forget that all measures, even those we habitually use without much forethought, have technical limitations and are valid only when assumptions about either the data or the processes that produced the data are met. If technical limitations are ignored or assumptions are not met, reliable conclusions cannot be drawn from the data. Problems can arise that are long-lived and difficult to overcome when inappropriate measures are repeatedly used within a research domain.

Correspondence should be addressed to K. Murnane, Department of Psychology, 3123G Zoology-Psychology Building, University of Maryland, College Park, MD 20742-4411 (e-mail: kmurnane@umd5.umd.edu).

-Accepted by previous editor, Geoffrey R. Loftus
Careful consideration of the limitations of measures and the assumptions on which measures rest is needed when memory for the source is investigated with the two-source, three-response-alternative task used by Henkel and Franklin (1998). In this task, information is learned from two sources, $A$ and $B$, and subjects have three response options: They can respond that a test item is old and came from Source A, that it is old and came from Source B, or that it is new. This type of memory test combines memory for the item (is the test item old or new?) and memory for the source (if the test item is old, is it from Source A or Source B?). The measurement problem is to disentangle memory for the item from memory for the source. Henkel and Franklin, along with several others (e.g., Batchelder \& Riefer, 1990), point out that many measures confound item and source memory under some circumstances. Murnane and Bayen (1996) evaluated a number of empirical measures of source memory and showed that most of these measures confound item and source memory most of the time.

Henkel and Franklin (1998) measure errors in source memory with a variant of what Murnane and Bayen (1996) called the conditional false source identification measure (CFSIM) for comparisons between two single sources. The difference between the variant of CFSIM used by Henkel and Franklin and the variant analyzed by Murnane and Bayen is that a critical independent variable, the nature of the similarity relationship between perceived and imagined items, is manipulated within sources in Henkel and Franklin's experimental design. In cases like this, performance (error rates in Henkel and Franklin's data) must be conditionalized on correct item recognition for each value of the independent variable separately for each source. Henkel and Franklin have done this, and the CFSIM vari- 
ant they use avoids most of the problems of confounding item and source memory that plague many commonly used measures of source memory.

If Henkel and Franklin (1998) have used the best available empirical measure, what is the cause of our concern? The main conclusion drawn by Henkel and Franklin is that memory for the source of an item can be affected by the properties of other items stored in memory. This conclusion rests on the finding that subjects tend to make more source memory errors for imagined items that are perceptually similar to items that have been perceived during learning than to imagined items that are either conceptually similar (in Experiment 1) or dissimilar to items that have been perceived during learning. This finding, if correct, has important implications for memory theories that attempt to explain source memory. For Henkel and Franklin's conclusion to be accepted, however, the increase in source memory errors they observed must not be caused by processes invoked to determine whether the test item was old or new.

Given that the variant of single-source CFSIM used by Henkel and Franklin (1998) is the best empirical measure of source memory available for their data, can the conclusion that perceptual similarity to other items in memory increases source memory errors be accepted without further consideration? Not if it is recognized that, like any measure, single-source CFSIM provides a valid measure of source memory only if certain theoretical assumptions are met. Consider the case in which the item memory process indicates that the test item is new and the source memory process indicates that the test item came from one of the sources in the experiment. How is a conflict like this resolved? Murnane and Bayen (1996, p. 426) showed that single-source CFSIM provides a measure of source memory that is unconfounded by item memory only if conflicts between the outputs from item and source memory processes are always resolved in favor of the output from item memory. In other words, if the item process indicates that the test item is new, the subject always responds "new" no matter how weak the evidence may be that the item is new, and no matter how compelling the evidence may be that the item came from one of the sources. Call this the recognition-dominance assumption. Murnane and Bayen showed that single-source CFSIM (along with every other empirical measure of source memory) confounds item and source memory if the recognitiondominance assumption is not met. Recognition dominance is a strong assumption. Because recognition dominance may not hold, it would be valuable to have additional evidence that Henkel and Franklin's pattern of errors was caused by source memory processes and not item memory processes.

Henkel and Franklin (1998) recognize this point, and they present further evidence in the form of both patterns in their data and a supplemental multinomial analysis in support of the claim that their measures of source memory are not confounded by item memory. We agree with Henkel and Franklin that additional analyses are needed to support this point. However, we believe that both the argu- ment they give concerning patterns in their data and the multinomial analyses they present have problems. These problems can be more clearly understood by recognizing the technical limitations of different measures and the theoretical assumptions on which the measures rest.

\section{Patterns in Item Recognition and Source Memory Data}

Henkel and Franklin (1998) argue that the source memory errors they observed cannot be based solely on differences in recognition memory between conditions because source memory and item memory were affected differently by the variables they manipulated. They provide examples in which source memory was worse in Condition A than in Condition $B$, whereas item recognition was either better in $A$ than in $B$ or was unaffected by the variable that differentiates Conditions A and B.

Although both plausible and compelling, this argument can be seen to be mistaken by noting technical characteristics of typical measures of item and source memory that are apparent in the mathematics used to compute each measure. Most of the measures that are commonly used to measure recognition performance are among the 10 measures of accuracy in a discrimination task analyzed by Swets (1986). Although Henkel and Franklin's (1998) measure of recognition performance (hits plus correct rejections) is not among those examined by Swets, it shares with all of Swets's measures the property of being based on performance for both old and new test items. In contrast, every empirical measure that conditionalizes measurement of source memory on correct item recognition is based only on performance for old items. An example is illustrated by the equation for the variant of single-source CFSIM for items from Source A used by Henkel and Franklin (a similar equation can be written for items from Source B):

$$
\text { single-source CFSIM }=\frac{Y_{A_{C} B}}{Y_{A_{C} A}+Y_{A_{C} B}},
$$

where $Y_{A C A}$ is the number of items from Source $\mathrm{A}$ in Condition $\mathrm{C}$ that the subject correctly identified as being from Source $\mathrm{A}$, and $Y_{A_{C} B}$ is the number of items from Source $\mathrm{A}$ in Condition $C$ that the subject incorrectly identified as being from Source B. All of the empirical measures of source memory identified by Murnane and Bayen (1996) that do not confound item and source memory if the recognition-dominance assumption is met measure source memory conditional on hits, and hence all of these measures are based only on performance for old items.

Because measures of item memory are based on performance for old and new items, whereas empirical measures of source memory are based only on performance for old items, measurement of item recognition is affected by a factor, performance on new items, that has no effect on measurement of source memory. Thus, measures of item recognition can vary independently of measures of source memory. The implications for drawing conclusions about whether a measure of source memory is confounded 


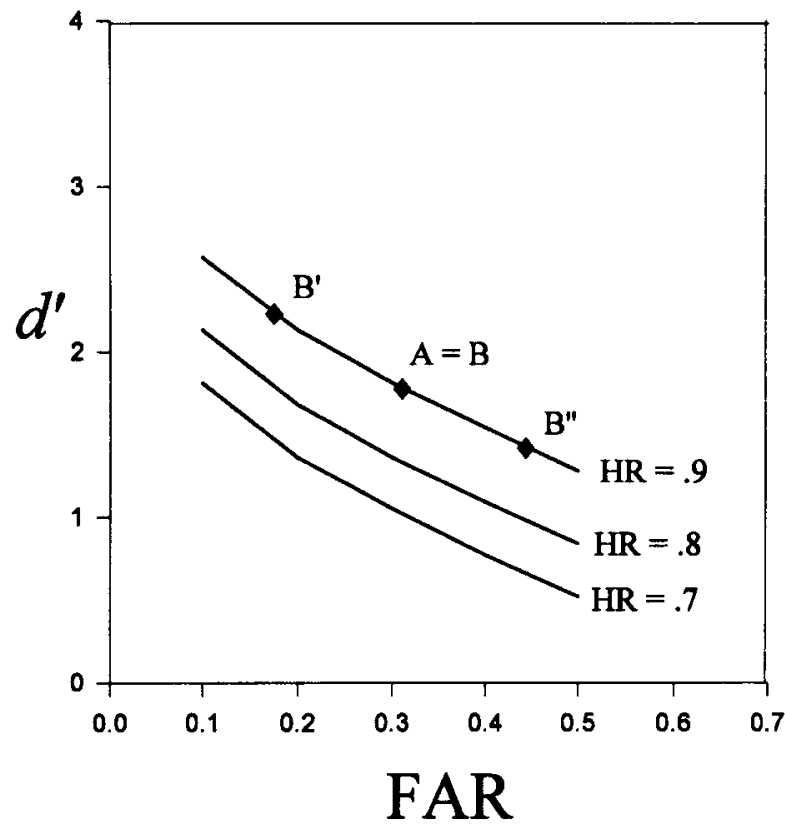

Figure 1. $d^{\prime}$ as a function of false alarm rate (FAR) for several different hit rates (HRs).

by item recognition from patterns of change in item and source memory across conditions of an experiment can be seen in Figure 1.

The figure shows $d^{\prime}$ as a function of false alarm rate (FAR) for several different hit rates (HRs). Suppose that the HRs for two conditions-say, A and B-of an experiment both equal .9. Suppose also that a higher proportion of the correctly recognized items have had their source correctly identified in Condition A than in Condition B. Possible $d^{\prime}$ values for the two conditions are labeled in the top function in the figure. If FARs in Conditions A and B are equal, item recognition will be the same in Conditions $A$ and $B$, while source memory will be higher in A than in B. If FARs are lower in Condition $\mathrm{B}^{\prime}$ than in Condition $\mathrm{A}$, item recognition will be higher in Condition $B^{\prime}$, while source memory will be higher in Condition A. Finally, if FARs are higher in Condition $\mathrm{B}^{\prime \prime}$ than in Condition $\mathrm{A}$, both item recognition and source memory will be higher in Condition A than in Condition B". Figure 1 shows that the same possibilities are present when HRs are not equal in Conditions $\mathrm{A}$ and $\mathrm{B}$. Thus, any pattern in item recognition across conditions in an experiment can be accompanied by any pattern in source memory across the same conditions. No reliable conclusions can be drawn about whether a particular measure of source memory is confounded with item memory by examining patterns of change in measures of item and source memory across conditions.

\section{Multinomial Analyses}

Several multinomial models of source memory are available in the literature (Batchelder \& Riefer, 1990; Batchelder, Riefer, \& Hu, 1994; Bayen, Murnane, \& Erd- felder, 1996). Multinomial models give unconfounded measures of item memory, source memory, and several types of response bias that can affect performance in a typical source memory task. For this reason, performance measures that are derived from multinomial models are sometimes preferred to empirical measures. Measurement using multinomial models is not assumption free, however. Multinomial models are members of the class of threshold decision theories, and their threshold assumptions have been the cause of recent controversy (Batchelder \& Riefer, 1990; Batchelder et al., 1994; Bayen et al., 1996; Kinchla, 1994).

Henkel and Franklin (1998) present multinomial analyses of their data using Batchelder and Riefer's (1990) onehigh threshold (1HT) multinomial model of source memory. They claim that the results of these analyses are "essentially equivalent" to the results of the analyses of variance (ANOVAs) performed on their empirical measures and that the multinomial analyses thus support the conclusion that the increase in source memory errors for test items that are perceptually similar to previously studied items was not caused by item memory processes. Again, however, there are technical considerations that must be taken into account when one is evaluating the results of the multinomial analyses. In some cases, these considerations invalidate the claim that the multinomial analyses support the conclusions drawn from the analyses based on empirical measures.

The full seven-parameter version of Batchelder and Riefer's (1990) 1HT model has two parameters that measure item memory, two parameters that measure source memory, and three parameters that measure response bias. This version of the model is unidentifiable using data from the typical two-source, three-response-alternative experimental paradigm because the data from this paradigm have only 6 df (Batchelder \& Riefer, 1990; Riefer, Hu, \& Batchelder, 1994). This problem may be solved by reducing the number of free parameters, which can be accomplished by imposing equality restrictions on the parameters. Batchelder and Riefer have identified a set of nested submodels using this technique and have presented a general step-by-step procedure for deciding which submodel to use (see, e.g., Bayen et al., 1996).

Henkel and Franklin (1998) have chosen Batchelder and Riefer's (1990) Model 6c to analyze their data, and, using the log-likelihood ratio statistic $G^{2}$ as a goodnessof-fit measure, they report that Model $6 \mathrm{c}$ provided a good fit to their data. They indicate that they chose Model $6 \mathrm{c}$ because they expected that item detection and source discrimination would differ for perceived and imagined items and because Model $6 \mathrm{c}$ includes freely varying parameters for both item and source memory for each source. Unfortunately, the choice of Model $6 \mathrm{c}$ weakens the support provided by the multinomial analyses for the conclusions reached on the analyses based on empirical measures. Model $6 \mathrm{c}$ is a saturated model with six free parameters and cannot be given an adequate goodness-of-fit test with a data set that has only $6 d f$. It is not surprising that Model $6 \mathrm{c}$ provided a "good fit" to Henkel and Franklin's data in al- 
most all experimental cells; the model has a free parameter for every degree of freedom in the data. Moreover, in their discussion of the problem of choosing an appropriate submodel, Batchelder and Riefer pointed out that Models $6 \mathrm{~b}$ and $6 \mathrm{c}$ cannot be differentiated with the data from an experiment that uses two sources. This means that analyses that use the 1HT multinomial model of source memory cannot determine whether the patterns of errors observed in Henkel and Franklin's data were caused by a source memory process or by a bias to respond that items had been previously perceived when the source cannot be remembered. This is a critical distinction in light of Henkel and Franklin's hypothesis that memory for the source of a test item is affected by other items in memory.

The degree of support provided by the multinomial analyses for the conclusions reached on the basis of the ANOVAs performed on empirical measures of source memory is also weakened by the data that were used in the multinomial analyses. Henkel and Franklin (1998) tested subjects with both targets and distractors that were similar to perceived items, similar to imagined items, or were not similar to any studied items. For reasons that are not made clear in the article, distractors, but not targets, were pooled across similarity conditions for the multinomial analyses. Pooling the distractors for the multinomial analyses is inappropriate because the source memory parameters in the 1HT multinomial model are sensitive to responses to distractor items. Depending on the pattern of false alarms across similarity conditions, unpooled distractor data can produce markedly different parameter values. Thus, pooling the distractor data makes it impossible to test any hypotheses about how source memory performance may change as a function of the similarity of perceived and imagined items, which is the main question of interest in Henkel and Franklin's study.

\section{Conclusion}

Henkel and Franklin (1998) present a series of welldesigned studies in support of the claim that memory for the source of an item can be affected by the characteristics of other items in memory. Their use of an appropriate em- pirical measure of source memory appears to provide good evidence in support of this claim. However, their empirical measure provides a measure of source memory that is unconfounded by item memory only if the recognitiondominance assumption is met. Recognition dominance is a strong assumption and therefore additional evidence in support of their main conclusion is desirable. Henkel and Franklin argue that patterns in the item and source memory data across conditions and a series of analyses using Batchelder and Riefer's (1990) multinomial model of source memory provide this additional evidence. However, neither the argument nor the multinomial analyses provide the desired support. The problems we have discussed in relation to the findings reported by Henkel and Franklin exemplify the general need for careful consideration of the technical characteristics of measures and the theoretical assumptions on which measures are based when one is conducting research on source memory.

\section{REFERENCES}

Batchelder, W. H., \& Riefer, D. M. (1990). Multinomial processing models of source monitoring. Psychological Review, 97, 548-564.

BatChelder, W. H., Riefer, D. M., \& Hu, X. (1994). Measuring memory factors in source monitoring: Reply to Kinchla. Psychological Review, 101, 172-176.

Bayen, U. J., Murnane, K., \& Erdfelder, E. (1996). Source discrimination, item detection and multinomial models of source monitoring. Journal of Experimental Psychology: Learning, Memory, \& Cognition, 22, 197-215.

Henkel, L. A., \& Franklin, N. (1998). Reality monitoring of physically similar and conceptually related objects. Memory \& Cognition, 26, 659-673.

KinchlA, R. A. (1994). Comments on Batchelder and Riefer's multinomial model of source monitoring. Psychological Review, 101, 166-171.

Murnane, K., \& Bayen, U. J. (1996). An evaluation of empirical measures of source identification. Memory \& Cognition, 24, 417-428.

Riefer, D. M., Hu, X., \& BaTChELDER, W. H. (1994). Response strategies in source monitoring. Journal of Experimental Psychology: Learning, Memory, \& Cognition, 20, 680-693.

SWETS, J. A. (1986). Indices of discrimination or diagnostic accuracy: Their ROCs and implied models. Psychological Bulletin, 99, 100-117.

(Manuscript received August 5, 1996; revision accepted for publication October 30, 1996.) 